\title{
Combining motivational and volitional approaches to reducing excessive alcohol consumption in pre-drinkers: a theory- based intervention protocol
}

\author{
Kim M. Caudwell, Barbara A. Mullan and Martin S. Hagger
}

\begin{abstract}
Background: Pre-drinking refers to the consumption of alcohol at home or a private residence prior to attending a subsequent social event. We present the study protocol of an online theory-based intervention to reduce predrinking and related harm in pre-drinking undergraduates, using behavior change techniques targeting the motivational and volitional phases of behaviour.

Design: A fully randomized 2 (autonomy support: present vs. absent) $\times 2$ (implementation intention: present vs. absent) between-participants design will be used to ascertain the effectiveness of the intervention in reducing predrinking alcohol consumption and alcohol-related harm. Participants will complete a range of theory-based measures prior to being allocated to one of the four experimental conditions. Four weeks later, participants will complete a follow-up questionnaire comprised of theoretical and behavioral measures.

Analyses: The main and interactive effects of the intervention components in reducing our primary dependent variables, namely, pre-drinking alcohol consumption and alcohol-related harm at four-week follow-up will be tested. Baseline alcohol consumption and demographic information will be included in the analysis as covariates.

Discussion: This online intervention is the first to be developed to reduce pre-drinking alcohol consumption, a behaviour linked to increased risk of alcohol-related harm. The intervention targets motivational and volitional components of the behaviour change process and is therefore likely to lead to greater reductions in pre-drinking alcohol consumption and experience of alcohol-related harm compared to either approach in isolation. If successful, the intervention can be implemented across various contexts and in populations where pre-drinking is prevalent.
\end{abstract}

Trial registration: ACTRN12614001102662. Registered 16 October 2014.

Keywords: Pre-drinking, Alcohol consumption, Theory-based intervention, Autonomy support, Implementation intention, Theoretical integration

\footnotetext{
* Correspondence:

Health Psychology \& Behavioural Medicine Research Group, School of

Psychology and Speech Pathology, Curtin University, Perth, Australia
} 


\section{Background}

Excessive alcohol consumption is associated with increased risk of acute (e.g., accidental injury) and chronic (e.g., cardiovascular disease, cancers, diabetes, liver disease, alcohol dependence, and a range of mental health conditions) harms [1]. In Australia, national costs of excessive alcohol consumption is estimated at 15 billion dollars annually, attributed to decreased workplace productivity, strain on the healthcare system, road or vehicular accidents, crime and associated costs, illness, and death [2]. Excessive alcohol consumption is especially apparent in university populations, with a third of students drinking to hazardous levels [3, 4] and appearing to outdrink their non-student peers on drinking occasions $[5,6]$. Research shows that excessive alcohol consumption significantly impairs students' health and academic performance, and increases risk-taking behaviors such as unplanned sexual activity [7].

Recent research has focussed on pre-drinking, the practice of consuming alcohol prior to attending a subsequent event, where alcohol consumption often continues [8, 9]. Pre-drinking is also referred to as prepartying [8], pregaming [9], and pre-loading [10]. Pre-drinking has been found to constitute more than $40 \%$ of alcohol consumption on drinking occasions [11], and an Australian multi-site study conducted in night entertainment areas found $65 \%$ of people reported pre-drinking prior to 'going out' for that evening [12]. Pre-drinking has been shown to be largely socially-motivated, with pre-drinkers citing "catching up" with friends and meeting new people as precipitating factors contributing to the popularity of these sessions [1315]. LaBrie et al. [15] found that interpersonal enhancement (i.e., pre-drinking for socialisation or enjoyment) was the strongest predictor of pre-drinking frequency and alcohol consumption, and demonstrated that pre-drinking motives differ from general alcohol consumption motives. Alcohol price has also been shown to be related to predrinking. Not only have students cited cost as influencing their pre-drinking [11, 16], but Miller and Droste [17] have shown that students change their hypothetical drinking decisions based on increases in the cost per drink. A recent study shows a relationship between strongly endorsing a cost motive for pre-drinking, and higher reported typical pre-drinking consumption [18].

In a series of recent studies, pre-drinking has been implicated as specifically contributing to alcohol-related harm. An event-level analysis by Barry et al. [19] found pre-drinking status significantly predicted blood-alcohol concentration, as measured by a breathalyser device. Merrill et al., [20] used event-level associations to reveal that pre-drinking on any given day was a significant predictor of alcohol related harm in university students, beyond both the total alcohol consumed on that day, and typical drinks consumed per day. In a sample of undergraduates, Caudwell and Hagger [18] found higher scores on pre-drinking cost motive items predicted higher incidence of alcohol-related harm in the previous twelve months. Pre-drinking appears to present an elevated risk to young adults, who demonstrate a lack of awareness of safe alcohol consumption limits [21], and, in laboratory settings, are unable to accurately pour a standard drink ${ }^{1}[22,23]$. To date, no interventions specifically aimed at reducing pre-drinking alcohol consumption have been developed. This protocol outlines a theory-based intervention that will attempt to reduce alcohol consumption during pre-drinking sessions, and the experience of alcohol-related harm.

\section{Theory-based interventions for excessive alcohol consumption}

One approach to reducing excessive alcohol consumption and alcohol-related harm among undergraduates is to develop behavioral interventions based on social psychological and motivational theories of health behavior. The use of such theories in informing interventions is important in targeting the influential determinants of health behavior, facilitating an understanding of "what works, and for whom", and allows for testing of the component theories in accounting for behavior change [24]. A range of health behavioral interventions targeting excessive alcohol consumption have been developed in university student populations, incorporating brief screening and feedback [25], motivational [26-28], peer or normative feedback [29-31], planning [32], and volitional approaches [33-35]. Though the efficacy of online interventions appears to bring about small changes in alcohol consumption behaviour $[d+=0.14$; [36]], many interventions are not theory-based, and there is evidence that theory-based interventions that closely develop intervention content to target specific psychological variables (commonly identified as correlates or predictors of alcohol consumption) are efficacious, with medium-sized effects [37, 38]. Furthermore, evidence supports the use of online delivery of alcohol interventions in student populations as they appear preferable to face-to-face methods (e.g., contact with a health professional) and may be especially useful for at-risk populations $[39,40]$. Therefore, the development of a theory-based online intervention to reduce pre-drinking alcohol consumption may be a useful endeavour.

\section{The theory of planned behavior}

The theory of planned behaviour [41] has been extensively applied to predict a range of health behaviours [42-44]. The theory considers behavioural intention the focal point of behavioural engagement, where intention is formed by belief-based constructs of attitude, subjective norm, and perceived behavioral control [41]. Attitude 
comprises belief-based evaluations of the behavior of interest; subjective norm consists of perceived social influence regarding behavioural engagement, and; perceived behavioral control constitutes the individual's ability to perform the behavior. The theory has been widely used across a range of health behavioural contexts, with a recent meta-analysis supporting the tenets of the theory-based model in predicting intention and behavior [44]. More recently, a meta-analysis of the theory applied to alcohol consumption behaviour has found attitudes strongly related to alcohol consumption intentions $\left(r_{+}=.62\right)$, and intentions moderately related to behaviour $\left(\mathrm{r}_{+}=.54\right)$ with authors concluding that both attitudes and intentions towards alcohol consumption are worthwhile targets for alcohol consumption behaviour change [45]. Generally, changes in behavioral intention appear to produce small-to-moderate changes in behaviour [46], with theory-based health behavioral interventions informed by the theory of planned behavior demonstrating particular efficacy $[\mathrm{d}+=0.36$; [36]], supporting our advocacy of adopting a theoretical approach.

A prominent criticism of the theory is the intention-behavior gap: the relative weakness in the link between intention and behaviour [47-50]. This is an important issue for interventions where intention may be the focus, yet it is a weak or modest predictor of behavioural engagement. For example, McEachan, Conner [44] shows the intention-behaviour relationship is weaker for health risk behaviours, such as abstaining from alcohol consumption, compared to health enhancing behaviors such as diet and exercise. A recent meta-analysis investigating the relationships between the theory of planned behaviour constructs applied to alcohol consumption concluded that interventions targeting attitudes and subjective norm may be worthwhile [45]. However, there is little utility in attempting to change intention through its antecedent constructs, where a substantial intention-behaviour gap is unlikely to facilitate meaningful behaviour change. This point and the utility of the theory of planned behaviour in health behavioural research is one of current debate (see [50]), with Schwarzer [51] suggesting that post-intentional (i.e., volitional) constructs that are known to influence behaviour are of importance in interventions based on the theory of planned behaviour. Implementation intentions [52] present an approach to "closing" the intention-behavior gap by linking important contextual cues to enacting the intended behaviour in the volitional stage, increasing the likelihood that the behavior is carried out in accordance with one's intentions.

\section{Implementation intentions and volition}

According to Gollwitzer [53], individuals who intend to reach an intended goal often fail to do so due to limitations in their ability to self-regulate behaviour. These limitations may constitute reasons such as failing to get started (e.g., forgetting or failing to act at the opportunity to do so) and getting derailed (e.g., due to attentional or competing factors; Gollwitzer \& Sheeran, [54]). For example, a pre-drinking goal intention may be "I intend to reduce my alcohol consumption drinking during predrinking sessions". However, an individual with this intention may not recognise the chance to enact that intention or fail to do so at the critical moment (e.g., where an environment is conducive to excessive alcohol consumption). Implementation intentions increase the likelihood that people will attain their intended goals by specifying contextual details of how these goals will be implemented, as well as when, and where [55]. An implementation intention for pre-drinking may therefore be "when I have finished an alcoholic drink at a pre-drinking session, I will then drink a glass of water or soft drink to help reduce my alcohol consumption". This allows individuals to switch from making conscious, effortful deliberations about enacting behaviour, to responding automatically to critical cues [52], mitigating the effects of self-regulatory limitations on carrying out intended behaviours. A meta-analysis by Gollwitzer and Sheeran [54] shows that there is a considerable effect $\left(d_{+}=.65\right)$ of implementation intentions in facilitating goal attainment over that of simply forming goal intentions. Importantly, implementation intention approaches have been shown to be effective in reducing alcohol consumption in young people including university students $[35,38,56]$.

Key features of an implementation intention approach include detailing how the intended behaviour will be enacted. In previous studies using this approach, participants either formed their own implementation intentions [38] or chose from a menu of responses to refusing a drink with the option of developing their own plan [35]. These studies and a recent review by Hagger and Luszczynska [57] suggest that implementation intentions may be more successful if they include additional planning components that address certain contingencies in an if-then format, such as "if I am offered an alcoholic drink, then I will politely refuse by saying, 'No thanks, I have to drive" [35]. In the context of pre-drinking, there are likely many contextual scenarios where individuals may be at risk of consuming excessive amount of alcohol (e.g., drinking games, coercion or pressure) $[9,58]$. Therefore, the formation of multiple implementation intentions to address these scenarios may be especially effective in reducing pre-drinking alcohol consumption. However, compelling individuals to intend to perform certain behaviours and assisting them in doing so may not be as effective if individuals lack the necessary motivational resources to facilitate the formation of these intentions and subsequent behavior. 


\section{Self-determination theory}

Another theoretical framework that has seen wide application in many health-related fields is self-determination theory [59-62]. Self-determination theory places the quality of an individuals' motivation as influential in behavioural engagement and persistence. Individuals who exhibit controlled motivation to engage in a behaviour tend to do so because of certain external contingencies - monetary incentive or reward, or for self-esteem rationales such as avoiding guilt or blame, or embarrassment [59]. Individuals who exhibit autonomous motivation to engage in a behaviour tend to do so because it serves personally-relevant goals or the act is itself intrinsically rewarding [59]. The more autonomously motivated an individual is towards engaging in behaviour, the more likely they will be to perform and persist in performing that behaviour [63, 64]. Recent evidence indicates attitudes and intentions towards engaging in health behaviour are more strongly linked to autonomous motivation rather than controlled motivation $[65,66]$.

Health behavioural interventions based on selfdetermination focus on the facilitation of autonomous motivation [60,67]. This is often achieved by providing autonomy support - a supportive context and rationale for the individuals' internalising of behavioural regulation [63]. The provision of autonomy support and facilitation of autonomous motivation have demonstrated validity in engendering positive behavioural change in a wide context of health behavioural settings [62]. Within the context of alcohol consumption, studies involving self-determination theory have found relationships between autonomous forms of motivation and reductions in self-reported alcohol consumption [68], as well as intentions to keep alcohol consumption within limits, and reductions in alcohol units consumed [69]. Pavey and Sparks found that autonomy in relation to perceptions of health risk information and autonomous motivation to engage in health protective behaviours were related to participation in those behaviours [70-72].

Conversely, studies on peer influences in college drinkers have shown individuals who exhibit controlled motivation to drink excessively do so because they tend to appraise situations from a controlled orientation, related to their sense of self-esteem [73]. Therefore, an intervention that provides an autonomy-supportive context for reduced alcohol consumption may prove effective for predrinkers who consume alcohol excessively or in contexts where motivation to reduce excessive alcohol consumption may be lacking. Given research demonstrating the importance of autonomy in enhancing receptiveness to health risk information, and indicating intrinsic goals are more likely to be pursued than those where individuals feel compelled to pursue goals [64, 70, 72], individuals may be more autonomously motivated to reduce their pre-drinking alcohol consumption if they generate their own autonomous reasons for pursuing such a goal.

\section{Evidence for combining approaches}

A meta-analysis of internet-based health behavioral interventions has found those incorporating more behavior change techniques tended to have larger effects, potentially due to these techniques targeting different components of the behaviour change process [36]. According to the model of action phases proposed by Heckhausen and Gollwitzer [74], a "Rubicon" exists between a deliberative, or predecisional phase, and a volitional, or preactional phase. The predecisional phase incorporates the feasibility and desirability of a behavioral outcome; the motivational tendency towards enacting that behavior which leads to the formation of a goal intention [75]. The preactional phase therefore incorporates how best to meet the behavioral goal - the stage at which individuals may fall short of meeting that goal due to limitations in their ability to self-regulate behavior [75]. It follows, therefore, that interventions targeting both motivation and volitional phases of action may be more effective in evoking behaviour change.

Studies have also shown that intentions are more likely to be carried out if they are formed consistent with autonomous reasons for engaging in the target behavior [76] and when the behavior is consistent with their psychological needs [77]. Evidence shows support for a synergistic relationship between autonomous motivation and the formation of implementation intentions in facilitating goaldirected behaviour. For example, a study on goal self-concordance (i.e., the extent to which a goal-directed behaviour is self-determined), found self-concordance significantly predicted progress on a range of participant goals, and that the relationship between goal self-concordance and progress was dependent on whether or not participants formed implementation intentions [78]. Koestner et al. [79] demonstrated that participants who formed autonomy-supportive implementation intentions achieved greater goal progress than those in a neutral condition $(d=.67)$. The authors attribute this to the internalisation of goals in a selfconcordant manner that reflects heightened personal interest and meaning. In terms of interventions based on this premise, targeting the motivational and volitional phases in tandem show increased efficacy in reducing alcohol consumption [33], promoting exercise behavior [80], reducing saturated fat intake [81], and improving fitness [82] over either approach in isolation.

\section{The present study}

The purpose of the present study is to test an online, theory-based intervention to reduce pre-drinking alcohol consumption among undergraduate students who pre-drink. The intervention will test the effects of two theory-based techniques targeting the predecisional and implemental phases of the model of action phases through: (1) facilitating autonomous motivation to 
reduce pre-drinking alcohol consumption, and (2) prompting the individual to form context-specific implementation intentions to help bridge the goal intention-behavior gap. Combining these techniques should see greater reductions in pre-drinking alcohol consumption and alcohol-related harm than either approach in isolation. The current research makes an original contribution to knowledge by adopting a factorial design, which permits us to examine the independent and interactive effects of two intervention components related to different processes in the model of action phases. The research builds on previous approaches to promoting autonomous motivation [79] and based on current 'best practice' recommendations for using implementation intentions [57]. It also follows on from research that suggests that incorporating both motivational and implemental phases is optimally effective in changing health behaviour by targeting multiple processes [38, 80, 81].

\section{Methods \\ Design}

The study will adopt a 2 (autonomy support: present vs. absent) x 2 (implementation intention: present vs. absent) design (see Fig. 1). Given evidence for the use of periodic prompts in supporting online interventions $[83,84]$ and the increased effectiveness of presenting reminders in implementation intention interventions [85] participants will be sent the components of their respective intervention via email following its conclusion. At follow-up, four weeks later, participants will be invited to complete the same theory-based measures as at baseline to assess the influence of the intervention in terms of changes in theoretical constructs and behavior.

\section{Intervention components}

Participants will be randomly allocated to one of four conditions: a control condition, an autonomy support condition, an implementation intention condition, and a combined autonomy support and implementation intention condition. Each condition will include the first two guidelines of the Australian National Health and Medical Research Council safe drinking guidelines [1]. These guidelines are recommendations for keeping alcohol consumption within limits to reduce the risk of alcohol-related harm over the lifetime, and are included in the Appendix. (Fig. 2) shows the intervention components alongside the intended action phase targets.

\section{Autonomy support condition}

Participants will be asked to generate statements that reflect a series of interpersonal conditions of autonomy support, as outlined in Su and Reeve [86]. These are

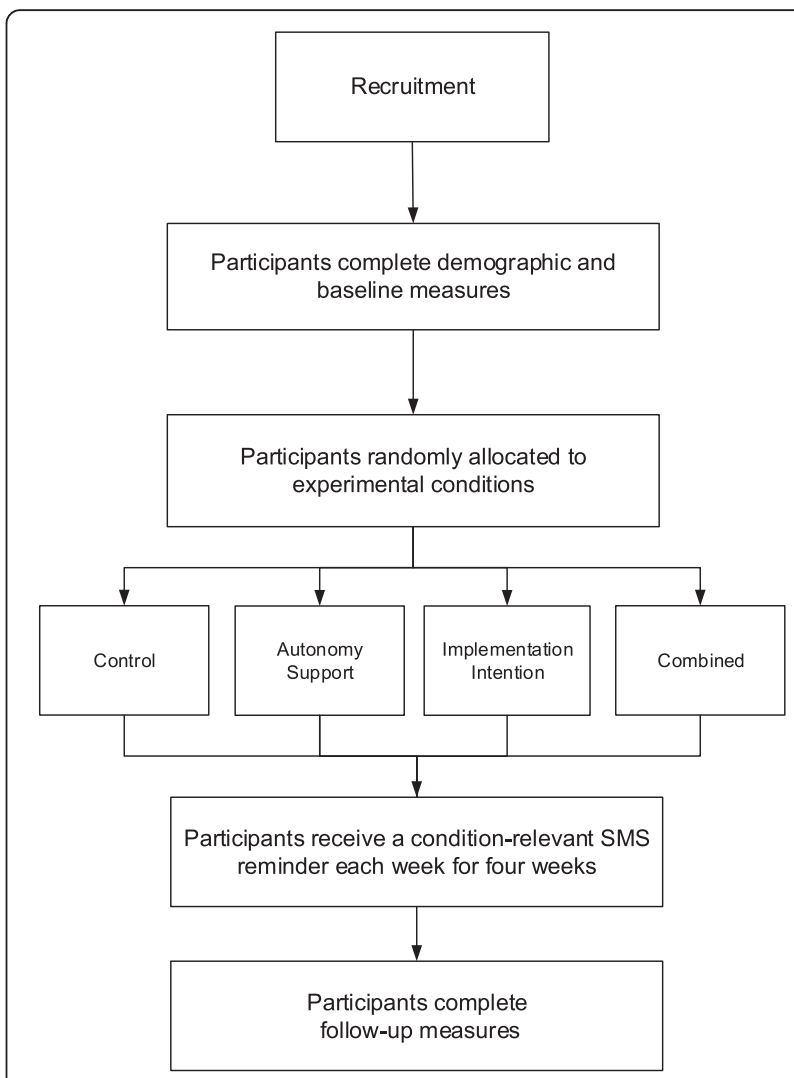

Fig. 1 Conceptual diagram showing the intervention components and their influence on the two stages of action [74]. ${ }^{1}$ National Health and Medical Research Council guidelines for reducing risk related to alcohol consumption

closely based on verified approaches used throughout self-determination theory-based interventions to facilitate autonomous motivation to engage in the target behavior [86-89]. The five conditions outlined in Su and Reeve [86] include: providing meaningful rationales (i.e., why self-regulated engagement in reducing pre-drinking alcohol consumption may be beneficial), acknowledging negative feelings (i.e., feelings associated with reducing pre-drinking alcohol consumption); use of noncontrolling language (e.g., may or could rather than must or should); offering choices (i.e., promoting choicemaking and encouragement), and nurturing inner motivational resources (i.e., making the satisfaction of needs for autonomy, competence, and relatedness salient in the communication). Table 1 includes example prompts and statements to be used in the intervention.

\section{Implementation intention condition}

Participants will be informed of how forming specific ifthen plans to reduce their alcohol intake during predrinking sessions can assist them in doing so. Given that personally-relevant goals have been found more effective in leading to behavioral engagement [90], and the 


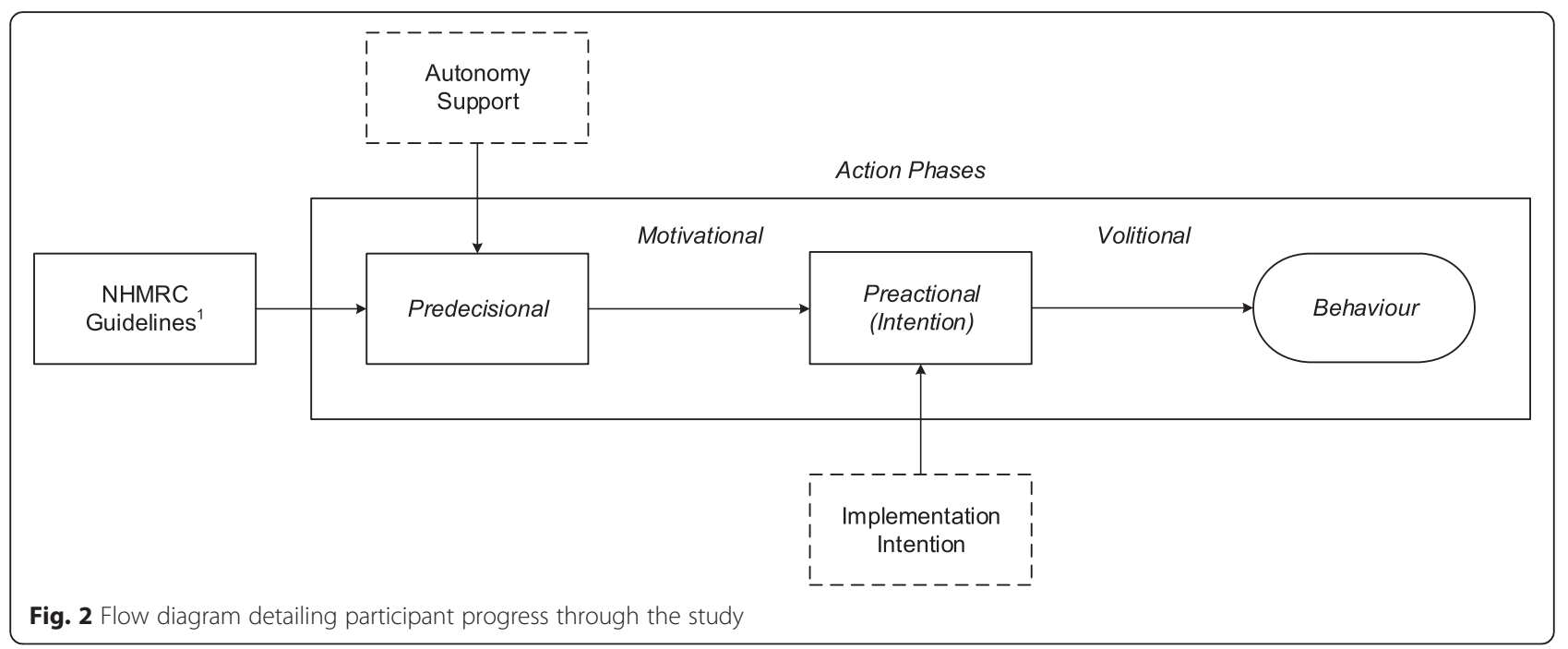

importance of self-relevant cues in leading to action, as outlined in Heckhausen and Gollwitzer [74], participants will be asked to detail a series of situations in which they might be at risk of excessive pre-drinking alcohol consumption. Participants will then be provided with examples of implementation intentions before being asked to generate their own that correspond to their identified situations, using two (i.e., if..., then...) open-response text boxes [57].

\section{Combined condition}

Heckhausen and Gollwitzer's [74] action-phase model places the predecisional (i.e., motivational) as preceding a behavioural decision, from which an individual passes through to the preactional (i.e., volitional) phase. Accordingly, participants in the combined condition will first receive the autonomy support component, followed by the implementation intention component. A conceptual map of the intervention components relative to the components in the action-phase model is included in Fig. 2.

\section{Measures}

\section{Theory of planned behaviour}

Attitude, subjective norm, and perceived behavioural control items will be used based on previous research [38, 91]. Attitude will be measured with a common item stem (i.e., "reducing alcohol consumption during predrinking sessions would be...") followed by a series of five bipolar adjectives (e.g., bad-good, beneficial-harmful), with participants asked to score each adjective accordingly on a six-point scale. Subjective norm will be measured with three statements referring to perceived pressure from others to engage in pre-drinking (e.g., "people who are important to me would want me to reduce my alcohol consumption during pre-drinking sessions") with participants asked to respond to each on six-point Likert-type scales ranging from 1 (strongly disagree) to 6 (strongly agree). Perceived behavioural control will be measured with three statements regarding control (e.g., If I wanted to, I could reduce my alcohol consumption during pre-drinking sessions), with participants asked to respond to each on six-point Likert-type scales ranging from 1 (strongly disagree) to 6 (strongly agree). Intention to reduce pre-drinking alcohol consumption will be measured with three items (e.g., I will reduce my alcohol consumption during pre-drinking sessions) with six-point Likert-type scales ranging from 1 (strongly disagree) to 6 (strongly agree).

\section{Planning}

Nine items from the planning subscale of the SelfRegulation Questionnaire [92] will be used to measure participants' planning ability. Participants will respond to these items (e.g., "I have trouble making plans to help me reach my goals") on six-point Likert-type scales ranging from 1 (strongly disagree) to 6 (strongly agree).

\section{Autonomous motivation and goal self-concordance}

Sheldon and Kasser [77] have developed a measure of goal self-determination, whereby participants rate how much they pursue goals for specific controlled, non-selfdetermined reasons (e.g., "...because somebody wants me to, or because I'll get something from somebody if I do", "I probably wouldn't do this if I didn't get some kind of reward, praise, or approval for it"), or autonomous, or selfdetermined reasons (e.g., because I really believe that it is an important goal to have - I endorse it freely and value it wholeheartedly). Participants will respond on nine-point Likert-type scales ranging from 1 (not at all for this reason) to 9 (completely because of this reason). Controlled scores are subtracted from autonomous scores to derive a relative score for goal self-concordance [76]. 


\section{Goal progress}

Participants will be asked to rate the extent of their progress, if any, in reducing their alcohol consumption during pre-drinking sessions, on a nine-point Likert-type scale ranging from 1 (none at all) to 9 (total progress), as used in previous research [78].

\section{Pre-drinking alcohol consumption}

Participants will report their pre-drinking alcohol consumption in terms of Australian standard drink equivalents consumed during pre-drinking sessions each week, over the previous four weeks, with the aid of a pictorial guide [1], at both baseline and follow-up. The pictorial guide comprises examples of typically served or available portion sizes of alcoholic beverages (e.g., a carton of beer, a bottle of wine or spirits) to aid in participant estimation of pre-drinking alcohol consumption (i.e., pre-purchased quantities such as bottles of spirits or cartons of beer). This approach has been used in previous research [91].

\section{Alcohol-related harm}

The Brief Young Adult Alcohol Consequences Scale (BYAACQ) [93] is a validated measure of the experience of alcohol-related harm that is well-suited to use in college populations for the purpose of evaluating change in alcohol consequences. The measure comprises a series of 24 participant-endorsed yes/no statements related to alcohol-related harm (e.g., "I have felt very sick to my stomach or thrown up after drinking"). Scores are derived from summing all yes responses to create a unidimensional index of alcohol-related harm [93]. Participants will complete the B-YAACQ at baseline and follow-up, to ascertain the effects of the intervention in reducing alcohol-related harm attributable to reductions in pre-drinking alcohol consumption. The time-frame of the B-YAACQ will be modified to refer to harm from alcohol consumption in the previous four-week period, to give a fine-grained view of the effects of the intervention on alcohol-related harm (see [94]).

\section{Hypotheses}

It is hypothesized that participants receiving both autonomy support and implementation intention components will exhibit greater reductions in pre-drinking alcohol consumption and alcohol-related harm at follow-up, relative to participants receiving either intervention component in isolation, in accordance with evidence supporting the combination of these approaches in potentially targeting two important components of the action-phase model $[64,75,76]$.

\section{Participants}

Eligible participants will be current undergraduate students who regularly consume alcohol (i.e., are current 'drinkers'), and have engaged in pre-drinking behaviour within the previous six months. Based on medium effects for implementation intentions on reductions in alcohol consumption reported in Hagger, Lonsdale [33] and the meta-analysis of self-determination theory applied to health contexts reported in Ng et al. [62], we conducted a statistical power analysis using $G^{*}$ Power to ascertain an adequate sample size for the intervention. Specifically, the power analysis was for an Analysis of Covariance (ANCOVA) on the two key dependent variables, alcohol consumption and summed B-YAACQ scores, with the intervention groups as the independent variables powered to detect a medium effect size (Cohen's $f=.25$ ) with power set at .80 and alpha set at .025 , and baseline scores on the dependent variable as a covariate. The analysis yielded 196 participants (i.e., 49 per group) for each analysis.

\section{Analyses \\ Randomisation check}

A 2 (autonomy support: present or absent) x 2 (implementation intention: present or absent) MANOVA will be conducted, with baseline demographic, behavioural, and psychological measures as dependent variables, and the intervention components as the independent variables, to test for between-group differences across the intervention conditions at baseline.

\section{Manipulation checks}

As the effect of implementation intentions might be diminished by participants failing to comply with instructions consistent with the approach, we will content analyse participants' implementation intention scripts (typed in response to the implementation intention manipulation) to ascertain the extent to which participants complied with the intervention instructions [33]. Independent raters familiar with implementation intentions will rate the quality of scripts based on the presence or absence of key planning components: (1) used the ifthen format, (2) specified a relevant, realistic, and appropriate cue, (3) linked the cue to the desired response. A one-way independent groups ANOVA will be conducted to test the effect of autonomy support on goal selfconcordance as a manipulation check.

\section{Effects of the intervention on pre-drinking alcohol consumption and alcohol-related harm}

Two ANCOVAs (autonomy support: present or absent) x 2 (implementation intention: present or absent) will be conducted to ascertain the effect of the intervention on followup self-reported pre-drinking alcohol consumption, and summed B-YAACQ scores, at follow-up, controlling for baseline pre-drinking alcohol consumption. 


\section{Effects of the intervention on psychological variables}

A 2 (autonomy support: present vs. absent) x 2 (implementation intention: present vs. absent) MANCOVA will be conducted, with autonomous motivation, constructs from the theory of planned behaviour (attitudes, subjective norm, perceived behavioural control, and intention) and goal progress as dependent variables, and pre-intervention pre-drinking alcohol consumption as a covariate.

\section{Ethics}

The study protocol was approved by Curtin University Research Ethics Committee (HR185/2014/AR1). Participants will provide informed consent to participate in the intervention.

\section{Discussion}

Pre-drinking is associated with significant risks attributable to excessive alcohol consumption [19, 20, 95]. No theory-based interventions to reduce pre-drinking alcohol consumption have yet to be developed. The present protocol has outlined a theory-based intervention that will attempt to reduce pre-drinking alcohol consumption and alcohol-related harm, by targeting the volitional and motivational phases of action, according to the actionphase model outlined by Heckhausen and Gollwitzer [74]. There is evidence that the provision of autonomy support is associated with greater autonomous motivation to engage in behaviour, and that autonomous motivations for reducing alcohol consumption are associated with reductions in alcohol consumption [33, 69]. Although, exhibiting motivation is a necessary but not sufficient condition for behavioural enactment $[49,96]$. Forming implementation intentions has been shown to strengthen the link between intention and behaviour, by providing a link between a contextual cue and an intended response $[35,54]$. Combining these approaches is based on the premise that promoting goal selfconcordance is important in successful goal attainment [78], and is integral to the efficacy of implementation intention approaches [57, 79]. Therefore, an approach that combines the volitional and motivational action phases, providing individuals with autonomy supportive context for behaviour change and the regulatory skills with which to translate this motivational impetus into behaviour, may be more effective in eliciting successful behaviour change. We therefore expect that while participants in the autonomy support and implementation intention conditions will report lower pre-drinking alcohol consumption at follow-up, the combination of these approaches will see the greatest reduction in pre-drinking alcohol consumption. This is because individuals may be autonomously motivated and intend to reduce their predrinking alcohol consumption, let may lack the regulatory capacity required to translate this intention into action (i.e., inclined abstainers) [48]. Similarly, the formation of if-then plans to reduce pre-drinking alcohol consumption may not lead to action if the underlying rationale for these plans is not autonomous [79]. Providing autonomy support to facilitate autonomous motivation will form a sound basis for the development of if-then plans, leading to the translation of that motivational basis into successful action and greater reductions pre-drinking alcohol consumption.

There are some limitations in the design of the intervention that should be noted. As the intervention will be delivered online, there may be potential problems with attrition between baseline and follow-up [33]. This has the potential to reduce the statistical power of the intervention to detect an effect, and limit testing intervention effects on the relevant theoretical constructs. To mitigate this, recruitment will attempt to account for the attrition rate observed in recent online interventions $[33,97]$. It is important to note that the primary focus of the intervention is to ascertain the overall efficacy of the intervention conditions in terms of reductions in the primary outcome variables, pre-drinking alcohol consumption and alcohol-related harm, rather than the mediating effects of theoretical constructs which are important issues but secondary to overall effects. Secondly, reviews of alcohol interventions often cite the lack of continued follow-up as detrimental to establishing the efficacy of these interventions over time [40, 98]. However, the efficacy of this intervention can be considered a basis for further research that ascertains the extent of intervention efficacy over time. Finally, there are many issues with the validity and accuracy of self-reported alcohol consumption [99]. However, by using pictorial aids detailing standard drink equivalents for commonlyconsumed alcoholic beverage containers [1], we attempt to mitigate errors in measurement. Further, by measuring goal attainment, the effect of the intervention on fulfilling participant goals to reduce pre-drinking alcohol consumption and alcohol-related harm can also be assessed.

\section{Endnotes}

${ }^{1}$ In Australia, a standard drink is defined as an alcoholic beverage containing $10 \mathrm{~g}$ of ethanol (alcohol).

\section{Appendix}

Intervention components

Control condition

Participants are presented with two guidelines from the NHMRC [1] to inform them of the relationship between alcohol consumption and the risk of alcohol-related harm over a lifetime, and reducing the risk of injury on a single occasion of drinking. 


\section{GUIDELINE 1}

Reducing the risk of alcohol-related harm over a lifetime.

The lifetime risk of harm from drinking alcohol increases with the amount consumed.

For healthy men and women, drinking no more than two standard drinks on any day reduces the lifetime risk of harm from alcohol-related disease or injury.

GUIDELINE 2

Reducing the risk of injury on a single occasion of drinking.

On a single occasion of drinking, the risk of alcoholrelated injury increases with the amount consumed.

For healthy men and women, drinking no more than four standard drinks on a single occasion reduces the risk of alcohol-related injury arising from that occasion.

\section{Autonomy support condition}

Participants are presented with a script comprising a series of statements using autonomy-supportive language, and given prompts to write about reasons pursuing the goal of reducing their pre-drinking alcohol consumption may be worthwhile:

"Over the next few weeks, we'd like you to consider reducing your pre-drinking alcohol consumption. Remember, when we talk about pre-drinking, we mean: drinking alcohol at home, or someone else's house, prior to attending an event (where drinking alcohol may continue).

Pre-drinking can be harmful, so there are many good reasons why people might set themselves the goal of reducing their pre-drinking alcohol consumption. While we understand that this goal may not be overly enjoyable or interesting, if you identify reasons why reducing your pre-drinking alcohol consumption is important to you, you may feel you are more able to meet this goal. You are free to choose exactly how you will reduce your predrinking alcohol consumption - developing your own strategy that uses your set of skills and resources often leads to success.

The following prompts are to help you think of ways you can reduce your pre-drinking alcohol consumption, avoid negative outcomes associated with alcohol consumption, and gain the benefits of reducing pre-drinking alcohol.

You will be taken through these ways step-by-step; as you read, you will be provided with reasons for completing these prompts and how they might help you.

Remember, whether or not you engage in these exercises is entirely up to you - it's your choice.

\section{Example:}

Identifying some of the negative consequences of predrinking can be a good first step in forming your plan to reduce your pre-drinking alcohol consumption. Reducing your alcohol consumption during pre-drinking sessions will help you to avoid some of these negative consequences you may experience when pre-drinking excessively."

\section{Implementation Intention Condition}

Participants are told they are more likely to reach their intended goal of reduced pre-drinking alcohol consumption if they think of "if-then plans" that specify when and where these plans will be enacted:

"You are more likely to carry out your intention to reduce the amount of alcohol you consume during predrinking sessions if you make a decision about the time and place you will do so, and how you plan to do it.

Decide now when and where you will need to limit the amount of alcohol you consume during pre-drinking sessions, and how you will do it. We want you to plan to reduce the pre-drinking alcohol you consume over the next month, paying particular attention to the specific situations in which you will need to implement these plans.

For example, you might find it useful to say to yourself: "When I finish an alcoholic beverage during a predrinking session, I will then drink a glass of water to help limit my alcohol consumption."

Alternatively, you might find it useful to say to yourself: "When I am offered a drink during a pre-drinking session, I will say, "No thanks, I have to get up early tomorrow."

\section{Example:}

Please choose from the options below, or write your plans in the text box available, following the format shown in the previous example (i.e., if... then...). Remember, it is important to remember the specific situation in which you need to implement your plan."

\section{Competing interests \\ The author(s) declare that they have no competing interests.}

\section{Authors' contributions}

$\mathrm{KC}$ and $\mathrm{MH}$ designed and developed the intervention and analytic plan, and KC drafted the manuscript. BM and $\mathrm{MH}$ contributed to the development of the intervention, and commented on subsequent versions of the manuscript. All authors read and approved the final manuscript.

\section{Acknowledgements}

None.

Received: 30 April 2015 Accepted: 18 December 2015

Published online: 16 January 2016

\section{References}

1. National Health and Medical Research Council. Australian guidelines to reduce risks from drinking alcohol. Canberra, ACT: Commonwealth of Australia; 2009. p. 179

2. Collins DJ, Lapsley HM. The avoidable costs of alcohol abuse in Australia and the potential benefits of effective policies to reduce the social costs of alcohol. In: Ageing DoHa. Canberra, ACT: Commonwealth of Australia; 2008.

3. Hallett J, Howat P, Maycock B, McManus A, Kypri K, Dhaliwal S. Undergraduate student drinking and related harms at an Australian university: web-based survey of a large random sample. BMC Public Health. 2012;12(1):37.

4. Todd J, Mullan B. Using the theory of planned behaviour and prototype willingness model to target binge drinking in female undergraduate university students. Addict Behav. 2011;36(10):980-6. 
5. Slutske WS. Alcohol use disorders among US college students and their non-college-attending peers. Arch Gen Psychiatry. 2005;62(3):321-7.

6. Slutske WS, Hunt-Carter EE, Nabors-Oberg RE, Sher KJ, Bucholz KK, Madden PAF, et al. Do college students drink more than their non-college-attending peers? Evidence from a population-based longitudinal female twin study. J Abnorm Psychol. 2004;113(4):530-40.

7. Wechsler H, Nelson TF. What we have learned from the Harvard School of Public Health College Alcohol Study: Focusing attention on college student alcohol consumption and the environmental conditions that promote it. J Stud Alcohol Drugs. 2008;69(4):481.

8. Pedersen ER, LaBrie J. Partying before the party: Examining prepartying behavior among college students. J Am Coll Heal. 2007;56(3):237-45. PubMed Language: English. Entry Date: 20080314. Revision Date: 20091218. Publication Type: journal article.

9. Borsari B, Boyle KE, Hustad JTP, Barnett NP, O'Leary Tevyaw T, Kahler CW. Drinking before drinking: Pregaming and drinking games in mandated students. Addict Behav. 2007;32(11):2694-705.

10. Reed MB, Clapp JD, Weber M, Trim R, Lange J, Shillington AM. Predictors of partying prior to bar attendance and subsequent BrAC. Addict Behav. 2011; 36(12):1341-3.

11. Read JP, Merrill JE, Bytschkow K. Before the party starts: risk factors and reasons for "pregaming" in college students. J Am Coll Heal. 2010;58(5):46172. PubMed Language: English. Entry Date: 20100507. Revision Date: 20110520. Publication Type: journal article.

12. Miller PG. Patron Offending and Intoxication in Night-Time Entertainment Districts (POINTED). Canberra: National Drug Law Enforcement Research Fund; 2013.

13. Hummer JF, Napper LE, Ehret PE, LaBrie JW. Event-specific risk and ecological factors associated with prepartying among heavier drinking college students. Addict Behav. 2013;38(3):1620-8.

14. Paves AP, Pedersen ER, Hummer JF, LaBrie JW. Prevalence, social contexts, and risks for prepartying among ethnically diverse college students. Addict Behav. 2012;37(7):803-10

15. LaBrie JW, Hummer JF, Pedersen ER, Lac A, Chithambo T. Measuring college students' motives behind prepartying drinking: Development and validation of the prepartying motivations inventory. Addict Behav. 2012;37(8):962-9.

16. Pedersen ER, LaBrie JW, Kilmer JR. Before you slip into the night, you'll want something to drink: exploring the reasons for prepartying behavior among college student drinkers. Issues Ment Health Nurs. 2009;30(6):354-63. PubMed Language: English. Entry Date: 20090814. Revision Date: 20110520. Publication Type: journal article.

17. Miller $\mathrm{PG}$, Droste N. Alcohol price considerations on alcohol and illicit drug use in university students. J Alcoholism Drug Dependence. 2013;1 (109):2.

18. Caudwell KM, Hagger MS. Pre-drinking and alcohol-related harm in undergraduates: the influence of explicit motives and implicit alcohol identity. J Behav Med. 2014;37(6):1252-62.

19. Barry AE, Stellefson ML, Piazza-Gardner AK, Chaney BH, Dodd V. The impact of pregaming on subsequent blood alcohol concentrations: An event-level analysis. Addict Behav. 2013;38(8):2374-7.

20. Merrill JE, Vermont LN, Bachrach RL, Read JP. Is the pregame to blame? Event-level associations between pregaming and alcohol-related consequences. J Stud Alcohol Drugs. 2013;74(5):757-64. PubMed PMCID: PMC3749319. Epub 2013/08/21. eng.

21. Livingston M. Perceptions of low-risk drinking levels among Australians during a period of change in the official drinking guidelines. Drug Alcohol Rev. 2012;31 (2):224-30.

22. White AM, Kraus CL, Flom JD, Kestenbaum LA, Mitchell JR, Shah K, et al. College students lack of knowledge of standard drink volumes: Implications for definitions of risky drinking based on survey data. Alcohol Clin Exp Res. 2005:29(4):631-8.

23. De Visser RO, Birch JD. My cup runneth over: Young people's lack of knowledge of low-risk drinking guidelines. Drug Alcohol Rev. 2012;31(2):206-12.

24. Michie S. What works and how? Designing more effective interventions needs answers to both questions. Addiction. 2008;103(6):886-7. PubMed Language: English. Entry Date: 20080822. Revision Date: 20110701. Publication Type: journal article.

25. Kypri K, Saunders JB, Williams SM, MCGee RO, Langley JD, Cashell-Smith ML, et al. Web-based screening and brief intervention for hazardous drinking: a double-blind randomized controlled trial. Addiction. 2004;99(11):1410-7.

26. Borsari B, Carey KB. Two brief alcohol interventions for mandated college students. Psychol Addict Behav. 2005;19(3):296.
27. Schaus JF, Sole ML, McCoy TP, Mullett N, O'Brien MC. Alcohol screening and brief intervention in a college student health center: a randomized controlled trial. J Stud Alcohol Drugs. 2009;16:131.

28. Kypri K, Hallett J, Howat P, McManus A, Maycock B, Bowe S, et al. Randomized controlled trial of proactive web-based alcohol screening and brief intervention for university students. Arch Intern Med. 2009;169(16):1508-14.

29. Walters ST, Vader AM, Harris TR. A controlled trial of web-based feedback for heavy drinking college students. Prev Sci. 2007;8(1):83-8. English.

30. Neighbors C, Larimer ME, Lewis MA. Targeting misperceptions of descriptive drinking norms: efficacy of a computer-delivered personalized normative feedback intervention. J Consult Clin Psychol. 2004;72(3):434.

31. Lewis MA, Neighbors C, Oster-Aaland L, Kirkeby BS, Larimer ME. Indicated prevention for incoming freshmen: Personalized normative feedback and high-risk drinking. Addict Behav. 2007;32(11):2495-508.

32. Black N, Mullan B. An intervention to decrease heavy episodic drinking in college students: The effect of executive function training. J Am College Health. 2014 (just-accepted):00-.

33. Hagger MS, Lonsdale A, Chatzisarantis NLD. A theory-based intervention to reduce alcohol drinking in excess of guideline limits among undergraduate students. Br J Health Psychol. 2012;17(1):18-43.

34. Armitage CJ. Effectiveness of experimenter-provided and self-generated implementation intentions to reduce alcohol consumption in a sample of the general population: a randomized exploratory trial. Health Psychol. 2009; 28(5):545.

35. Murgraff $V$, White $D$, Phillips $K$. Moderating binge drinking: It is possible to change behaviour if you plan it in advance. Alcohol Alcohol. 1996;31(6):577-82.

36. Webb TL, Joseph J, Yardley L, Michie S. Using the internet to promote health behavior change: A systematic review and meta-analysis of the impact of theoretical basis, use of behavior change techniques, and mode of delivery on efficacy. J Med Internet Res. 2010;12(1):e4.

37. Hagger MS, Lonsdale A, Chatzisarantis NLD. Effectiveness of a brief intervention using mental simulations in reducing alcohol consumption in corporate employees. Psychol Health Med. 2011;16(4):375-92.

38. Hagger MS, Lonsdale AJ, Koka A, Hein V, Pasi H, Lintunen T, et al. An intervention to reduce alcohol consumption in undergraduate students using implementation intentions and mental simulations: A cross-national study. Int J Behav Med. 2012;19(1):82-96.

39. Kypri K, Saunders JB, Gallagher SJ. Acceptability of various brief intervention approaches for hazardous drinking among university students. Alcohol Alcohol. 2003;38(6):626-8.

40. White AW, Kavanagh D, Stallman H, Klein B, Kay-Lambkin F, Proudfoot J, et al. Online alcohol interventions: A systematic review. J Med Internet Res. 2010;12(5):e62.

41. Ajzen I. The theory of planned behavior. Organ Behav Hum Decis Process. 1991;50(2):179-211.

42. Armitage CJ, Conner M. Efficacy of the theory of planned behaviour: A meta-analytic review. Br J Soc Psychol. 2001;40:471-99. PubMed PMID: 219214254; 11795063. English.

43. Conner M, Sparks P. Theory of planned behaviour and health behaviour. In: Conner M, Norman P, editors. Predicting health behaviour. 2nd ed. Berkshire: McGraw-Hill Education; 2005. p. 170-222.

44. McEachan RRC, Conner M, Taylor NJ, Lawton RJ. Prospective prediction of health-related behaviours with the theory of planned behaviour: A metaanalysis. Health Psychol Rev. 2011;5(2):97-144.

45. Cooke R, Dahdah M, Norman P, French DP. How well does the theory of planned behaviour predict alcohol consumption? A systematic review and meta-analysis. Health psychology review. 2014 (ahead-of-print):1-20.

46. Webb TL, Sheeran P. Does changing behavioral intentions engender behavior change? A meta-analysis of the experimental evidence. Psychol Bull. 2006;132(2):249.

47. Sheeran P. Intention-behavior relations: A conceptual and empirical review. Eur Rev Soc Psychol. 2002;12(1):1-36.

48. Orbell S, Sheeran P. 'Inclined abstainers': A problem for predicting healthrelated behaviour. Br J Soc Psychol. 1998;37(2):151-65.

49. Rhodes RE, Bruijn GJ. How big is the physical activity intention-behaviour gap? A meta-analysis using the action control framework. Br J Health Psychol. 2013;18(2):296-309.

50. Sniehotta FF, Presseau J, Araujo-Soares V. Time to retire the theory of planned behaviour. Health Psychology Review. 2013:1-8

51. Schwarzer R. Life and death of health behaviour theories. Health Psychol Rev. 2013;8(1):53-6. 
52. Gollwitzer PM, Brandstätter V. Implementation intentions and effective goal pursuit. J Pers Soc Psychol. 1997;73(1):186.

53. Gollwitzer PM. Goal achievement: The role of intentions. Eur Rev Soc Psychol. 1993:4(1):141-85

54. Gollwitzer PM, Sheeran P. Implementation intentions and goal achievement: A meta-analysis of effects and processes. Adv Exp Soc Psychol. 2006;38:69-119.

55. Webb TL, Sheeran P. How do implementation intentions promote goal attainment? A test of component processes. J Exp Soc Psychol. 2007;43(2): 295-302.

56. Murgraff $V$, Abraham C, McDermott M. Reducing Friday alcohol consumption among moderate, women drinkers: Evaluation of a brief evidence-based intervention. Alcohol Alcohol. 2007:42(1):37-41.

57. Hagger MS, Luszczynska A. Implementation intention and action planning interventions in health contexts: State of the research and proposals for the way forward. Applied Psychology: Health and Well-Being. 2014;6(1):1-47.

58. Polizzotto MN, Saw MM, Tjhung I, Chua EH, Stockwell TR. Fluid skills: drinking games and alcohol consumption among Australian university students. Drug Alcohol Rev. 2007;26(5):469-75.

59. Ryan RM, Connell JP. Perceived locus of causality and internalization: Examining reasons for acting in two domains. J Pers Soc Psychol. 1989; 57(5):749-61.

60. Deci EL, Ryan RM. Self-determination theory: A macrotheory of human motivation, development, and health. Can Psychol. 2008;49(3):182-5.

61. Vallerand RJ, Pelletier LG, Koestner R. Reflections on self-determination theory. Can Psychol. 2008;49(3):257-62.

62. Ng JYY, Ntoumanis M, Thøgersen-Ntoumani C, Deci EL, Ryan RM, Duda JL, et al. Self-determination theory applied to health contexts. Perspect Psychol Sci. 2012;7(4):325-40.

63. Pelletier LG, Fortier MS, Vallerand RJ, Briere NM. Associations among perceived autonomy support, forms of self-regulation, and persistence: A prospective study. Motiv Emot. 2001;25(4):279-306.

64. Vansteenkiste M, Simons J, Lens W, Sheldon KM, Deci EL. Motivating learning, performance, and persistence: the synergistic effects of intrinsic goal contents and autonomy-supportive contexts. J Pers Soc Psychol. 2004; 87(2):246.

65. Hagger MS, Hardcastle SJ, Chater A, Mallett C, Pal S, Chatzisarantis NLD. Autonomous and controlled motivational regulations for multiple healthrelated behaviors: between- and within-participants analyses. Health Psychol Behav Med. 2014;2:565-601.

66. Caudwell KM, Hagger MS. Predicting alcohol pre-drinking in Australian undergraduate students using an integrated theoretical model. Applied Psychology: Health and Wellbeing. in press:1-26.

67. Ryan RM, Patrick H, Deci EL, Williams GC. Facilitating health behaviour change and its maintenance: Interventions based on self-determination theory. Eur Health Psychol. 2008;10(1):2-5.

68. Keatley DA, Clarke DD, Hagger MS. The predictive validity of implicit measures of self-determined motivation across health-related behaviours. $\mathrm{Br}$ J Health Psychol. 2013;18(1):2-17.

69. Hagger MS, Lonsdale AJ, Hein V, Koka A, Lintunen T, Pasi H, et al. Predicting alcohol consumption and binge drinking in company employees: An application of planned behaviour and self-determination theories. $\mathrm{Br} \mathrm{J}$ Health Psychol. 2011;17:1-29.

70. Pavey LJ, Sparks P. Reactance, autonomy and paths to persuasion: Examining perceptions of threats to freedom and informational value. Motiv Emot. 2009;33(3):277-90.

71. Pavey LJ, Sparks P. Autonomy and defensiveness: Experimentally increasing adaptive responses to health-risk information via priming and selfaffirmation. Psychol Health. 2011;27(3):259-76.

72. Pavey LJ, Sparks P. Autonomy and reactions to health-risk information. Psychol Health. 2010;25(7):855-72. PubMed Language: English. Entry Date: 20100910. Revision Date: 20110909. Publication Type: journal article.

73. Neighbors C, Larimer ME, Markman Geisner I, Knee CR. Feeling controlled and drinking motives among college students: Contingent self-esteem as a mediator. Self Identity. 2004;3(3):207-24.

74. Heckhausen H, Gollwitzer PM. Thought contents and cognitive functioning in motivational versus volitional states of mind. Motiv Emot. 1987;11(2):10120. English.

75. Achtziger A, Gollwitzer PM. Motivation and volition in the course of action. In: Heckhausen J, Heckhausen H, editors. Motivation and Action. Cambridge: Cambridge University Press; 2008. p. 272-95.
76. Sheldon KM, Elliot AJ. Not all personal goals are personal: Comparing autonomous and controlled reasons for goals as predictors of effort and attainment. Pers Soc Psychol. 1998;24(5):546.

77. Sheldon KM, Kasser T. Pursuing personal goals: Skills enable progress, but not all progress is beneficial. Personal Soc Psychol Bull. 1998:24(12):1319-31.

78. Koestner R, Lekes N, Powers TA, Chicoine E. Attaining personal goals: Selfconcordance plus implementation intentions equals success. J Pers Soc Psychol. 2002;83(1):231-44

79. Koestner R, Horberg E, Gaudreau P, Powers T, Di Dio P, Bryan C, et al. Bolstering implementation plans for the long haul: The benefits of simultaneously boosting self-concordance or self-efficacy. Personal Soc Psychol Bull. 2006;32(11):1547-58

80. Milne S, Orbell S, Sheeran P. Combining motivational and volitional interventions to promote exercise participation: Protection motivation theory and implementation intentions. Br J Health Psychol. 2002;7(2):163-84.

81. Prestwich A, Ayres K, Lawton R. Crossing two types of implementation intentions with a protection motivation intervention for the reduction of saturated fat intake: A randomized trial. Soc Sci Med. 2008;67(10): 1550-8.

82. Prestwich $\mathrm{A}$, Lawton $\mathrm{R}$, Conner $\mathrm{M}$. The use of implementation intentions and the decision balance sheet in promoting exercise behaviour. Psychol Health. 2003;18(6):707-21.

83. Fry JP, Neff RA. Periodic prompts and reminders in health promotion and health behavior interventions: systematic review. J Med Internet Res. 2009; 11(2):e16. PubMed PMCID: Pmc2762806. Epub 2009/07/28. eng.

84. Sainsbury K, Mullan B, Sharpe L. A randomized controlled trial of an online intervention to improve gluten-free diet adherence in celiac disease. Am J Gastroenterol. 2013;108(5):811-7.

85. Sniehotta FF, Scholz U, Schwarzer R, Fuhrmann B, Kiwus U, Völler H. Longterm effects of two psychological interventions on physical exercise and self-regulation following coronary rehabilitation. Int J Behav Med. 2005;12(4): 244-55.

86. Su Y-I, Reeve J. A meta-analysis of the effectiveness of intervention programs designed to support autonomy. Educ Psychol Rev. 2011;23(1): 159-88. PubMedacademic_MSTAR_854116002. English.

87. Cheon SH, Reeve J, Moon IS. Experimentally based, longitudinally designed, teacher-focused intervention to help physical education teachers be more autonomy supportive toward their students. J Sport Exerc Psychol. 2012; 34(3):365-96.

88. Cheon SH, Reeve J. Do the benefits from autonomy-supportive PE teacher training programs endure?: A one-year follow-up investigation. Psychol Sport Exer. 2013;14(4):508-18.

89. Hagger MS, Chatzisarantis NLD. The trans-contextual model of autonomous motivation in education: Conceptual and empirical issues and meta-analysis. Rev Educ Res. 2015. Available at: http://rer.sagepub.com/content/early/2015/ 05/13/0034654315585005.abstract

90. Adriaanse MA, de Ridder DTD, de Wit JBF. Finding the critical cue: Implementation intentions to change one's diet work best when tailored to personally relevant reasons for unhealthy eating. Personal Soc Psychol Bull. 2009;35(1):60-71.

91. Caudwell KM and Hagger MS. Predicting alcohol pre-drinking in Australian undergraduate students using an integrated theoretical model. Applied Psychol: Health and Well-Being, 2015;7(2):188-213.

92. Brown JM, Miller WR, Lawendowski LA. The self-regulation questionnaire. 1999.

93. Kahler CW, Hustad J, Barnett NP, Strong DR, Borsari B. Validation of the 30-day version of the Brief Young Adult Alcohol Consequences Questionnaire for use in longitudinal studies. J Stud Alcohol Drugs. 2008;69(4):611-5. PubMed PMCID: PMC2493538. Epub 2008/07/10. eng.

94. Kuntsche E, Labhart F. Drinking motives moderate the impact of predrinking on heavy drinking on a given evening and related adverse consequences_-an event-level study. Addiction. 2013;108(10):1747-55.

95. Labhart F, Graham K, Wells S, Kuntsche E. Drinking before going to licensed premises: An event-level analysis of predrinking, alcohol consumption, and adverse outcomes. Alcohol Clin Exp Res. 2013;37(2):284-91.

96. Orbell S, Sheeran P. Motivational and volitional processes in action initiation: A field study of the role of implementation intentions. J Appl Soc Psychol. 2000;30:780-97.

97. Postel MG, de Haan HA, Ter Huurne ED, Becker ES, de Jong CA. Effectiveness of a web-based intervention for problem drinkers and reasons for dropout: randomized controlled trial. J Med Internet Res. 2010;12(4):e68. 
98. Carey KB, Scott-Sheldon LAJ, Carey MP, DeMartini KS. Individual-level interventions to reduce college student drinking: A meta-analytic review. Addict Behav. 2007;32(11):2469-94.

99. Del Boca FK, Darkes J. The validity of self-reports of alcohol consumption: State of the science and challenges for research. Addiction. 2003;98:1-12.

Submit your next manuscript to BioMed Central and we will help you at every step:

- We accept pre-submission inquiries

- Our selector tool helps you to find the most relevant journal

- We provide round the clock customer support

- Convenient online submission

- Thorough peer review

- Inclusion in PubMed and all major indexing services

- Maximum visibility for your research

Submit your manuscript at www.biomedcentral.com/submit

Biomed Central 